\title{
Rentenprivatisierung in Bismarck-Ländern: Zur Rolle der Sozialpartner als Vetospieler
}

\author{
Tobias Wiß
}

\begin{abstract}
Pension Privatization in Bismarckian Countries: The Role of Social Partners as Veto Players Abstract: The article analyses the role of social partners within the process of changing pension systems in four countries: Germany, Austria, Belgium, and Italy. So far the research has focused on partisan actors and public pensions, whereas this contribution extends the analysis to non-state actors (social partners) and to non-state social policy (occupational pensions). Only the extension and improvement of the theories concerning veto players and veto points allow for the inclusion of the political-parliamentary arena and the collective arena in order to capture the overall power and role of social partners. The main result is, that far-reaching pension reforms in Bismarckian countries with multiple veto possibilities can be observed if governments win social partners over with package deals and if social partners can expect new future (veto) power.
\end{abstract}

Keywords: Pension Reforms, Privatization, Social Partners, Veto Players, Bismarckian Countries

Schlagwörter: Rentenreformen, Privatisierung, Sozialpartner, Vetospieler, Bismarck-Länder

\section{Einleitung ${ }^{1}$}

Alternde Gesellschaften, zurückgehende Geburtenraten sowie finanzielle Engpässe üben einen kontinuierlichen Druck auf europäische Sozialsysteme aus und machen Reformen notwendig. Vor allem konservative Wohlfahrtsregime stehen einer Reformsklerose gegenüber, werden diese doch mit Attributen wie „Reformstau“ und „frozen landscapes“ (Esping-Andersen 1996), also mit trägen und unbeweglichen Institutionen, charakterisiert. Der locus classicus von Wohlfahrtsregimen, die Alterssicherung, erscheint hierbei als das am schwierigsten zu reformierende Politikfeld, da es den größten Ausgabenanteil des Sozialbudgets ausmacht, Übergänge von umlagefinanzierten Systemen zu kapitalfundierten Modellen aufgrund des Doppelzahlerproblems² für unwahrscheinlich erachtet werden und zudem starke, auf den Status-quo gerichtete Interessen vor allem der Gewerkschaften vorhanden sind (Pierson 1996, 1998). Für Reformen oder gar einen Wandel der Alterssicherung müssen erhebliche Hürden in Form von institutionellen Vetopunkten über-

1 Der Artikel entstand innerhalb des DFG-geförderten Projektes „Regulierung von Zusatzrenten in Europa“ (EB 434/1-2). Ich danke Bernhard Ebbinghaus und den beiden anonymen Gutachterinnen/Gutachtern für hilfreiche Kommentare.

2 Dieses Problem ergibt sich aus der Situation, dass Beitragszahler bei einem Übergang zu kapitalgedeckten Systemen einerseits Beiträge zum alten Umlagesystem entrichten müssen, um die heutigen Renten zu finanzieren. Gleichzeitig sind diese aber auch dazu aufgefordert, Beiträge für ihre eigene spätere Rente zu zahlen, um so Kapital zu akkumulieren. 
wunden werden. Dennoch hat Wandel stattgefunden, in den meisten europäischen Ländern sind in den vergangenen zwei Jahrzehnten zum Teil erhebliche Reformdynamiken auszumachen. Vor allem für konservative Wohlfahrtsregime war dies nicht zu erwarten, gleichwohl haben sich auch diese Länder als recht beweglich und für Wandel zugänglich gezeigt (Palier 2010; Ebbinghaus 2011).

Wieso waren Reformen in diesen Ländern möglich und welchen Einfluss haben die Sozialpartner im politischen Gesetzgebungs- und Implementierungsprozess? Zur Beantwortung dieser Frage erfolgt a) die Erweiterung des Vetospieleransatzes, b) Verfeinerung des Vetopunkteansatzes und c) Berücksichtigung der politischen und kollektiven Ebene bzw. der Politikgestaltung und Implementierung/ Selbstregulierung. Es wird die Hypothese überprüft, dass weitreichende Rentenreformen auch in Ländern mit vielen Vetomöglichkeiten zu beobachten sind. Sozialpartner als zentrale Vetoakteure in Bismarck-Ländern können vor allem dann für Reformen gewonnen werden, wenn Paketlösungen vereinbart werden und die Sozialpartner mit einer starken künftigen Vetorolle rechnen können.

Generell sind zwei Arenen für Rentenreformen entscheidend, die parteipolitische Arena (Regierungsparteien und Oppositionsparteien) und die korporatistische Arena (Regierung und Sozialpartner) (Bonoli 2000; Schludi 2005; Natali u. Rhodes 2008). Vor allem in Ländern mit kooperativen Formen von Sozialpartnerschaft ist die zweite Ebene elementar (Pierson 1998, S. 556f; Visser u. Hemerijck 1998), da Konfliktlinien in Bismarck-Ländern stärker zwischen politischen Akteuren und Sozialpartnern als zwischen den Parteien verlaufen (Bonoli 2000; Natali u. Rhodes 2008, S. 28, 42). Während bei bisherigen Analysen zu Rentenreformen auf parteipolitische Akteure und staatliche Rentensysteme fokussiert wurde (Huber u. Stephens 2001; Kitschelt 2001; Schludi 2005), erfuhren nicht-staatliche Akteure wie die Sozialpartner und nicht-staatliche Sozialpolitik wie die betriebliche Altersvorsorge eine weniger systematische Berücksichtigung.

In seiner Studie zu staatlichen Rentenreformen kommt Marier (2008) zu dem Ergebnis, dass die Beziehungen von Staat und Sozialpartnern zusammen mit dem Wahlsystem erklärungsrelevante Faktoren sind. Nicht so sehr die Anzahl von Vetospielern als vielmehr deren Natur ist für Erfolg und Scheitern von Reformen wesentlich. Ähnlich führt die Analyse von Béland (2001) zu dem Ergebnis, dass Gewerkschaften bei Rentenkürzungen durch ihre Rolle als Anbieter tarifvertraglicher Renten als formaler oder ideologischer Vetoakteur fungieren. Zudem kann Arbeitgeberverbänden ein Interesse an sozialpolitischen Leistungen unterstellt werden, da auch die Wirtschaft vom Sozialstaat zu profitieren scheint (Hall u. Soskice 2001; Mares 2004; Swenson 2004). Im Bereich der bAV weisen neuere Studien auf die wachsende Bedeutung von Zusatzrenten hin (Hacker 2005; Trampusch 2007; Ebbinghaus 2011). Hier knüpft die vorliegende Studie an und vereint die beiden Forschungsperspektiven: Der Einbezug von Implementierung und Selbstregulierung (bAV) ist, zusätzlich zur Politikgestaltung (staatliche Rente), zentral, um die gegenwärtige Altersversorgungspolitik sowie die gesamt Macht der Sozialpartner zu verstehen. Bisherige Analysen räumen zwar die Möglichkeit von Reformpaketen auf staatlicher Ebene ein, bspw. die stärkere Berücksichtigung von Kindererziehungszeiten bei der Rentenberechnung im Austausch für Rentenkürzungen. Machtpolitisch erfahren die Sozialpartner hierdurch aber keinen Gewinn. Dies ist nur bei 
Einbezug der kollektiven Ebene ersichtlich, wenn z. B. den Sozialpartnern in der bAV mehr Verantwortung und damit Macht übertragen wird.

Ziel dieser Studie ist die Analyse der Sozialpartner und deren Beitrag zum Wandel der Alterssicherung in konservativen Wohlfahrtsregimen. Ein besseres Verständnis von Wandel trotz zahlreicher Vetospieler und Vetopunkte kann durch die Ergänzung der Vetospieler um nicht-staatliche Akteure (Sozialpartner) und durch den Einbezug der kollektiven Ebene, auf der Sozialpartner eigenständig über nicht-staatliche Sozialpolitik (bAV) entscheiden, erreicht werden. Hierbei wird über die bisherige Literatur hinausgegangen, die entweder die kollektive Ebene vernachlässigt oder aber getrennt von der parlamentarischen Ebene betrachtet hat. Diese Lücke wird geschlossen, indem beide Ebenen Eingang in die Analyse finden und Verbindungslinien aufgezeigt werden, die für mögliche Kompensationen und Reformpakete auch aus machtpolitischer Perspektive wichtig sind. Reformen und Wandel finden zwar maßgeblich über Parteien und Parlamente statt, allerdings können die Sozialpartner in diesen Prozess gestalterisch intervenieren. Weiterhin muss die anschließende Phase der Implementierung einbezogen werden, um ein vollständigeres Bild von Wandel und Reformen zu erhalten. Entscheidend für Reformen sind das Zusammenwirken und die Verbindungslinien von parlamentarisch-politischer und kollektiv-sozialpartnerschaftlicher Arena.

Anstelle nach Unterschieden in verschiedenen Typen von Ländern mit verschiedenen Rentensystemen zu suchen, zielt dieser Artikel auf eine systematische vergleichende Analyse von Bismarck-Ländern ab, um so mittels der Untersuchung von Fällen, die eher schlechte Voraussetzungen für eine Theorie - hier BismarckSysteme und Wandel trotz vieler Vetospieler - bieten, möglichst große Unterstützung für die Weiterentwicklung der entsprechenden Theorien zu erhalten. Ähnliche Reformdynamiken in ähnlicher institutioneller Umwelt werden mit der analytischen Verfeinerung der Theorien zu Vetospielern und Vetoansätzen abgeglichen. Zur Untersuchung werden politischen Prozesse (politische Arena) und die Ausgestaltung der betrieblichen Altersvorsorge (kollektive Arena) in vier Ländern herangezogen - Deutschland, Österreich, Belgien und Italien. Alle vier Länder können als Konsensdemokratien und konservative Wohlfahrtsregime im Bereich Renten charakterisiert werden. Trotz der für diese Länder typischen Pfadabhängigkeiten und hohen Hürden des Wandels sind in den letzten beiden Jahrzehnten Reformdynamiken auszumachen. ${ }^{3}$ Weiterhin ist die starke Rolle der Sozialpartner bei der Ausgestaltung der Alterssicherung in allen Ländern zentral, so dass deren gesamte Vetomacht nur unter Einbezug der bAV (Implementierung/Selbstregulierung) bestimmt werden kann. Länderübergreifende Gemeinsamkeiten und Unterschiede werden an fallinternen Feststellungen bemessen (Collier 1993, S. 115-116; Mahoney 2003, S. 360-361). Mittels pattern matching, der Identifikation von Mustern, werden die einzelnen Muster der vier Länder in der politischen und der kollektiven Arena verglichen, um so die theoretischen Erwartungen aufgrund der Erweiterung von Vetospielern und Vetopunkten zu überprüfen und die Prozesse des Wandels der Alterssicherung in Bismarck-Ländern unter Berücksichtigung der Sozialpartner

3 Aufgrund von Eigenschaften typisch für Mehrheitsdemokratien findet Frankreich als Land mit einem Bismarck-Rentensystem im Folgenden keine Berücksichtigung. 
auf Gemeinsamkeiten hin zu untersuchen. Untersuchungsgegenstand sind hierbei einzelne Reformen, wobei solche mit und ohne gewerkschaftlichen Einbezug Eingang finden. Zwar vermag diese Vorgehensweise nicht sämtliche Reformprozesse zu erklären, dennoch kann so ein besseres Verständnis über Reformaktivitäten trotz multipler Vetomöglichkeiten erreicht werden.

Hierzu wird zunächst Bezug zu Theorien über Vetospieler und Vetopunkte genommen, da Rentenreformen und Wandel durch individuelle und kollektive Entscheidungsträger herbeigeführt werden, was wiederum vom institutionellen Kontext abhängig ist. Daran anknüpfend wird auf die wesentlichen Charakteristika der untersuchten Länder bezüglich Rentensystem und Sozialpartnerschaft eingegangen. Hierauf aufbauend erfolgt im ersten Schritt die Analyse des Politikgestaltungsprozesses (politische Arena) und der Beteiligung der Sozialpartner (de facto Vetospieler). Grundannahme ist, dass der Einfluss der Sozialpartner von institutionellen Konfigurationen abhängt und somit ähnliche Konfigurationen auch einen ähnlichen Einfluss hervorrufen. Entsprechend dem Argument, dass Reformen mit Unterstützung der Sozialpartner vor allem bei einem Ausbau von Zusatzrenten, welche diesen zusätzliche Machtressourcen verschaffen, möglich sind, fokussiert der zweite Teil auf die tarifvertraglichen Zusatzrenten und die hier neu geschaffenen Gestaltungsspielräume (kollektive Ebene und formale Vetospieler).

\section{Nicht-staatliche Akteure und Politikebenen bei Reformen: Ein Analysemodell}

Um den institutionellen Rahmen für politische Aktivitäten und den Einfluss von Sozialpartner auf Rentenreformen abzustecken, wird zunächst Bezug auf das politische System genommen, bevor dann anschließend durch Berücksichtigung von Vetospielern und Vetopunkten die Akteursebene stärker in den Vordergrund rückt.

\subsection{Bismarck-Länder und Konsensdemokratien}

Die Art der Demokratie liefert wichtige Hinweise dafür, wie der Gesetzgebungsprozess organisiert ist und welche Institutionen und Akteure bei der Politikgestaltung berücksichtigt werden müssen. Den vier Bismarck-Ländern ist gemeinsam, dass sie Konsensdemokratien nach Lijphardt (1999) sind, in denen eingeschränkte Handlungsspielräume von Regierungen den Sozialpartnern Einfallstore für Einfluss öffnen. Konsensdemokratien lassen sich hierbei durch Mehrparteiensysteme, Koalitionsregierungen, Machtbalance der Exekutive, Verhältniswahlsysteme, Zweikammersysteme, korporatistische Interessengruppen, föderalen Staatsaufbau und rigider Verfassung(sgerichtsbarkeit) definieren. Insgesamt konkretisieren diese politischen Systemstrukturen die Beteiligung von Institutionen und Akteuren an der Politikgestaltung und weisen diesen Blockademöglichkeiten zu. Deutschland, Österreich, Belgien und Italien fallen bei Berücksichtigung der für die Verabschiedung von Rentenreformen relevanten Dimension Exekutive-Parteien sowie beim Indikator Zweikammersystem in die Gruppe der Konsensdemokratien, auch wenn die Länderkammer in Österreich (Bundesrat) nur aufschiebende Vetomacht besitzt. 
Der notwendige Einbezug mehrerer Institutionen und Akteure erweitert zugleich die Suche nach Legitimationsressourcen, so dass die Verantwortung für mit Kürzungen einhergehende Maßnahmen auf mehrere Schultern verteilt und damit Legitimität erhöht wird (Myles u. Pierson 2001). Die Zuordnung von Verantwortung und Gefahr einer Bestrafung von Rentenkürzungen durch Abwahl der Regierung ist in Ländern mit Einparteienregierungen und Einkammersystemen höher als in Ländern mit Koalitionsregierungen und Zweikammersystemen. Daher kann vermutet werden, dass Mehrheitsdemokratien einschneidende Reformmaßnahmen eher scheuen, während hingegen in Konsensdemokratien höhere Reformaktivitäten erwartet werden können.

\subsection{Vetospieler und Vetopunkte}

Im Gegensatz zu bisherigen Analysen, welche sich auf die staatliche Ebene beschränken, ist für Reformprozesse aufgrund möglicher Paketlösungen und Kompensationen auch die nicht-staatliche Ebene zu berücksichtigen. Dies erfolgt durch Erweiterung des Vetospieler- und des Vetopunkteansatzes. Indem die kollektive Ebene und die dortige Vetopunkte Eingang in Analysen finden, müssen auch die dortigen Vetospieler erfasst werden, um diese für Reformen zu gewinnen. Die Ansätze zu Vetospielern (Tsebelis 1995, 2002) und Vetopunkten (Immergut 1992; Bonoli 2001) knüpfen an die Lijphart'sche Unterscheidung von Mehrheits- und Konsensdemokratie an (Kaiser 1998), indem beide Ansätze die für die Verabschiedung von Gesetzen benötigten Institutionen und Akteure berücksichtigen. Weiterhin schließen sich die Konzepte von Vetospielern und Vetopunkten nicht gegenseitig aus, sondern ergänzen sich vielmehr.

Grundgedanke des Vetospielertheorems ist, dass je höher die Anzahl von parteipolitischen und institutionellen Vetospielern sowie deren ideologische Distanz sind, desto höher ist politische Stabilität und desto geringer sind Reformen und Wandel (Tsebelis 2002). Generell lassen sich parteipolitische und institutionelle Vetospieler unterscheiden (Tsebelis 2002). In Koalitionsregierungen, typisch für Konsensdemokratien, sind in der Regel mehrere Parteien an der Regierung beteiligt. Je nach ideologischer Distanz stellt jede an der Regierung beteiligte Partei einen Vetospieler dar, da diese bei Gesetzesvorhaben berücksichtigt werden müssen. Institutionelle Vetospieler sind je nach Zusammensetzung und Ausgestaltung Verfassungsgerichte und Parlamentskammern. Je nach Land, Art des Gesetzes und Zusammensetzung der Parlamente sind Regierungen in Zweikammersystemen bei der Verabschiedung von Gesetzen auf zwei Parlamentskammern angewiesen und müssen zudem auf die Vereinbarkeit mit der Verfassung achten (Verfassungsgericht). Der Status der zweiten Kammer als Vetospieler hängt dabei gemäß der Absorptionsregel von der Akteursbesetzung ab. In der Regel verfügen die Regierungsparteien über die Mehrheit in der ersten Parlamentskammer, diese bildet also keinen Vetospieler. Sollte die Opposition über die Mehrheit in der zweiten Kammer verfügen und ist deren Zustimmung zu Gesetzen notwendig, bildet die zweite Kammer einen Vetospieler. Haben die Regierungsparteien auch hier die Mehrheit, entfällt der Status eines Vetospielers. 
Allerdings zeigen jüngere Studien, dass eine hohe Anzahl von Vetospielern nicht zwingend $\mathrm{zu}$ mehr Stabilität und verringerten Reformaktivitäten führt. Machtteilung aufgrund von Vetospielern und hohe Reformaktivitäten schließen sich nicht aus (Lindvall 2010, S. 360). Erfolgreiche Rentenreformen hängen weniger stark von der Anzahl der Vetospieler ab, als vielmehr von Paketlösungen (quid pro quo) und geeigneten Koalitionen, so dass potenzielle Vetospieler zur Zustimmung bewogen werden können (Bonoli 2000, S. 172-173; Häusermann 2010; Lindvall 2010). Mit der Anzahl der Vetospieler nimmt die Reformaktivität sogar zu (Immergut u. Abou-Chadi 2010). Je schwieriger Reformen aufgrund von Blockademöglichkeiten durchzusetzen sind, desto mehr Möglichkeiten und Angriffspunkte ergeben sich gleichzeitig für die Sozialpartner, Einfluss geltend zu machen. Als Lösungsmöglichkeit für diesen Widerspruch bietet sich die Inklusion von Nebenschauplätzen an. Mittels Einbezug der Implementierungsphase und der Selbstregulierung der bAV können Verbindungslinien und Kompensationsmöglichkeiten zwischen politischer und kollektiver Ebene gezogen und damit ein Beitrag zu Reformaktivitäten geleistet werden.

Um auch die Bedeutung von Sozialpartnern innerhalb von politischen Prozessen erfassen zu können, wird im Folgenden eine dritte Art von Vetospielern integriert, die ideellen/de facto/vorschlagsberechtigten Vetospieler (Héritier 2001; Orenstein 2008, S. 56-57). Dieser Typus, im Folgenden de facto Vetospieler genannt, zeichnet sich aus durch einflussreiche Akteure, die zwar formell auf staatlicher Ebene nicht berücksichtigt werden müssen, für eine erfolgreiche Umsetzung von Politik jedoch deren Einbezug in den Reformprozess empfohlen wird bzw. in der Regel erfolgt. Durch diese Konstruktion können auch nicht-staatliche Akteure wie Sozialpartner Einzug finden. So lassen sich die formalen Vetospieler, die Gesetze verabschieden und verhindern können, von de facto Vetospielern unterscheiden. Letztere können einerseits über verschiedene Kanäle argumentativ und beratend Einfluss auf politische Akteure und Gesetzesinhalte nehmen und bspw. mit Reformvorschlägen und Änderungswünschen an Politiker, Parteien und Ministerien herantreten. Andererseits besitzen die Sozialpartner in der Implementierungsphase und in ihrer Eigenschaft als Tarifvertragsparteien Vetomacht in der kollektiven Arena. Gewerkschaften und Arbeitgeberverbände sind hier bei Rentenreformen in Bismarck-Ländern zentral, da diese die Hauptbeitragszahler der staatlichen Rente repräsentieren, in die Verwaltung der Rentensysteme involviert sind und zudem über Handlungsspielräume in der Tarifpolitik verfügen, die sich generell für Nebenabsprachen eignet. Da sie aber Gesetzesvorhaben formal nicht blockieren können besitzen sie de facto Vetomacht. Insgesamt stehen sich somit parteipolitische (Regierungsparteien, Mehrheit in der zweiten Parlamentskammer), institutionelle (Parlamentskammern und Verfassungsgerichte) sowie de facto (Sozialpartner) Vetospieler gegenüber.

Um nun neben den nicht-staatlichen Akteuren auch die nicht-staatliche Ebene und die Implementierungsphase für die Analyse der Sozialpartner bei Rentenreformen nutzbar zu machen und so die gesamte Vetomacht zu bestimmen, wird das Konzept der Vetopunkte verfeinert. Vetopunkte, die zur Blockade des politischen Prozesses genutzt werden können, bilden strategische Kontexte für politische Akteure und dienen diesen als Einfallstore für die Beeinflussung der Politikgestaltung 
(Kaiser 1998, S. 529). Erzeugt werden Vetopunkte, verstanden als Gelegenheitsstrukturen der Einflussnahme, durch den Kontext des politischen Systems. Im Gegensatz zu Vetospielern beziehen sich Vetopunkte a priori nicht auf bestimmte Akteure. Vielmehr können sich verschiedene Akteure dieser institutionellen Angriffspunkte bemächtigen, um Reformprozesse zu blockieren bzw. zu beeinflussen (Immergut 1992). Generell ist der „Kundenkreis“ von Vetopunkten höher als beim Vetospieleransatz, was der Analyse der Rolle von Sozialpartnern förderlich ist. In Bezug auf Lijphart zeichnen sich Konsensdemokratien (bzw. Verhandlungsdemokratien) durch zahlreich vorhandene Vetopunkte aus (Kaiser 1998, S. 537). Allerdings lässt die klassische Version von Vetopunkten lediglich Aussagen über die Politikgestaltung zu. Dabei wird innerhalb des politischen Prozesses die Implementation von Gesetzen und Möglichkeiten der Selbstregulierung außer Acht gelassen, obwohl gerade diese Ebene Nebenabsprachen und Paketlösungen ermöglicht bzw. Reformen bei der Implementierung ver- und behindert werden können.

Gewerkschaften und Arbeitgeber können ihre Interessen und damit Einfluss an politischen und kollektiven Vetopunkten zum Ausdruck bringen. Vetopunkte der Konkordanz (Wahlsystem, Regierungssystem, Ministerien) und legislative Vetopunkte (Parlamentskammern, hohe Hürden für Verfassungsänderungen) bilden zusammen politische Vetopunkte, während sich kollektive Vetopunkte aus den Vetopunkten der Delegation (quasi-staatliche Behörden wie die Selbstverwaltung von Sozialversicherungen, Tarifautonomie, tripartistische Gremien) und der Expertise (Schlichtungsbehörden) zusammensetzen (Kaiser 1998). Politische Einflussmöglichkeiten bieten sich den Sozialpartnern sowohl auf staatliche als auch betriebliche Rentensysteme. Durch die Mitwirkung bei der Ausarbeitung von Reformentwürfen bei Ministerien sowie durch zum Teil persönliche Verbindungen zu Parteien und Parlamenten können diese ihre Interessen im politischen Prozess zum Ausdruck bringen. Zusätzlich können die Sozialpartner aber auch an kollektiven Vetopunkten Macht ausüben. Im Bereich der staatlichen Rentensysteme sitzen die Sozialpartner in der Selbstverwaltung und sind verantwortlich für die Implementation von Reformen. Bei den betrieblichen Rentensystemen ist die Aushandlung und Umsetzung von Tarifverträgen und der bAV direkt von den Sozialpartnern und Kollektivverhandlungen abhängig, ebenso wie der Aufbau und Organisation von paritätisch organisierten Zusatzrentenversorgungswerken.

Zusammengefasst veranschaulicht Tabelle 1 die Erweiterungen und Zusammenführung von Vetospielern und Vetopunkten. Durch Inklusion von de facto Vetospielern und kollektiven Vetopunkten kann der analytische und der gesamte machtpolitische Rahmen abgesteckt werden, innerhalb dessen die Sozialpartner an der Ausgestaltung der Alterssicherung und Rentenreformen partizipieren können. In der politischen Arena, der formalen Politikgestaltung, beeinflussen die Sozialpartner als de facto Vetospieler durch Interessenartikulation und Kraft der Argumente die Vetopunkte Regierung, Parlamente und Ministerien, ebenso wie durch ihre Rolle in der Umsetzung von Reformen sowie ihrer Stellung in der kollektiven Arena. Die Zustimmung von de facto Vetospielern zu Reformvorhaben kann vor allem dann erreicht werden, wenn diese erwarten, auch in Zukunft Vetomacht zu besitzen (Lindvall 2010, S. 361, 374). In der kollektiven Arena, bei der Implementierung von Gesetzen und Selbstregulierung der bAV, treten Sozialpartner dagegen als for- 
male Vetospieler an kollektiven Vetopunkten auf, indem die Selbstverwaltung der staatlichen Renten sowie der Abschluss von Tarifverträgen zur bAV und die Errichtung von gemeinsamen Versorgungswerken deren zwingende Zustimmung benötigt. Folglich haben die Sozialpartner umso mehr Einfluss auf der politischen Bühne, je mehr Entscheidungsmacht sie in der bAV und innerhalb der Selbstverwaltung der staatlichen Rentenversicherung haben, welche diese dann wiederum argumentativ auf politischer Ebene benutzen können. Je mehr die Sozialpartner in der politischen Arena verlieren, desto wichtiger wird für sie die kollektive Ebene. Hierdurch können Antworten auf die Frage nach der Rolle der Sozialpartner bei Rentenreformen und Möglichkeiten zur Kompensation gegeben werden, was für die Erklärung von Reformen trotz multipler Vetomöglichkeiten zentral ist.

Tabelle 1: Die Sozialpartner als Vetospieler an Vetopunkten in der Alterssicherung

\begin{tabular}{|l|l|l|l|}
\hline \multicolumn{2}{|c|}{} & Vetospieler & Vetopunkt \\
\hline \multirow{2}{*}{$\begin{array}{l}\text { Politische } \\
\text { Arena }\end{array}$} & $\begin{array}{l}\text { staatliche } \\
\text { Rentensysteme }\end{array}$ & de facto & $\begin{array}{l}\text { politisch } \\
\text { (Gesetzgebung, Parlament, Ministerien) }\end{array}$ \\
\cline { 2 - 4 } & $\begin{array}{l}\text { betriebliche } \\
\text { Rentensysteme }\end{array}$ & de facto & $\begin{array}{l}\text { politisch } \\
\text { (Gesetzgebung, Parlament, Ministerien) }\end{array}$ \\
\hline \multirow{2}{*}{$\begin{array}{l}\text { Kollektive } \\
\text { Arena }\end{array}$} & $\begin{array}{l}\text { staatliche } \\
\text { Rentensysteme }\end{array}$ & formal & $\begin{array}{l}\text { kollektiv } \\
\text { (Selbstverwaltung) }\end{array}$ \\
\cline { 2 - 4 } & $\begin{array}{l}\text { betriebliche } \\
\text { Rentensysteme }\end{array}$ & formal & $\begin{array}{l}\text { kollektiv } \\
\text { (Tarifverträge, kollektive Versorgungswerke) }\end{array}$ \\
\hline
\end{tabular}

Quelle:vgl. Wiß 2011, S. 50.

\section{Rentensysteme und Sozialpartnerschaft in Bismarck-Ländern}

Inwieweit weisen die vier Länder zentrale Eigenschaften von Bismarck-Systemen auf und welche Macht haben die Sozialpartner? Ein Blick auf die Rentensysteme zeigt starke Ähnlichkeiten, während die Sozialpartner zum Teil leicht unterschiedlich organisiert sind.

\subsection{Rentensysteme}

Allen Ländern gemeinsam sind zentrale Charakteristika von Bismarck-Rentensystemen: Die Alterssicherung wird durch Arbeitgeber und Arbeitnehmer über Sozialversicherungsbeiträge basierend auf dem Umlageverfahren finanziert und von diesen auch organisiert und verwaltet (Selbstverwaltung). In der Regel sind Leistungen einkommensbezogen (Äquivalenzprinzip) und sollen den im Erwerbsleben erreichten Lebensstandard aufrecht erhalten. Dementsprechend gering war in der Vergangenheit die Notwendigkeit, zusätzlich eine betriebliche oder individuelle Altersvorsorge abzuschließen.

In Deutschland sind alle wichtigen Gremien der Selbstverwaltung paritätisch besetzt. In Österreich dagegen besteht die Selbstverwaltung der Pensionsversiche- 
rung zu zwei Dritteln aus Vertretern der Versicherten und nur zu einem Drittel aus Vertretern der Arbeitgeber. Die frühere Vormachtstellung der Versicherten im die einzelnen Sozialversicherungsträger koordinierenden Hauptverband wurde durch die jüngsten Reformen durch eine paritätische Zusammensetzung abgelöst. Das zentrale Gremium der Sozialversicherungen in Belgien ist das Landesamt für Soziale Sicherheit (RSZ), welches gemeinsam von Gewerkschaften, Arbeitgebern und vom Staat geführt wird. Der subsidiäre Verwaltungsrat des Landespensionsamtes (RVP) besteht aus je sieben Gewerkschafts- und Arbeitgebervertretern und zwei staatlich Delegierten (De Deken 2011, S. 62). Die wesentlichen Institutionen in Italien sind das Rentensystem für Beschäftigte in der Privatwirtschaft (INPS) und das Rentensystem für Beschäftigte im öffentlichen Sektor (INPDAP). Ähnlich wie in Deutschland sind die Arbeitgeber zusammen mit den Gewerkschaften in die Verwaltung eingebunden. Die Rolle der Sozialpartner innerhalb der Selbstverwaltung ist teilweise unterschiedlich organisiert, allen Ländern gemeinsam sind aber die nur geringen Handlungsbefugnisse, bestehend aus der Satzung und Organisation sowie der Umsetzung gesetzlicher Vorgaben und Regelungen. Keinen formalen Einfluss hat die Selbstverwaltung auf Beitragssätze und Leistungshöhe.

Aufgrund der unterschiedlichen Beitragsbemessungsgrenzen und Rentenniveaus sind Zusatzrenten heute insgesamt für Deutschland und Belgien von höherer Bedeutung als für Österreich und Italien, wo auch Beschäftigte mit höheren Einkommen ihre Lebensstandardsicherung nur aus der staatlichen Rente beziehen können (siehe Tabelle 2). Weiterhin weisen Italien und Österreich höhere Beitragssätze auf als Deutschland und Belgien.

\section{Tabelle 2: Beitragsbemessungsgrenze, Rentenniveau und Beitragssatz 2010}

\begin{tabular}{lllll}
\hline & \multicolumn{2}{l}{ Beitragsbemessungsgrenze } & Nettorentenniveau & Beitragssatz \\
\cline { 2 - 4 } & \multicolumn{2}{c}{$\begin{array}{l}\text { absolut (EUR) } \\
\text { Deinkommens }\end{array}$} & $58,4 \%$ & \\
\cline { 2 - 4 } Beutschland & $66.000^{1}$ & 154 & $66,0 \%$ & $19,9 \%$ \\
Belgien & 46.895 & 118 & $89,9 \%$ & $16,4 \%$ \\
Österreich & 55.020 & 142 & $76,2 \%$ & $22,8 \%$ \\
Italien & 88.669 & 337 & $32,7 \%$ \\
\hline
\end{tabular}

Anmerkungen: '1 nur West, BBG Ost: 55.800. Quellen: OECD 2011a, S. 111, 125, 153.

\subsection{Sozialpartnerschaft und politisches System}

In Deutschland bilden die beiden parteiübergreifenden Dachverbände DGB (Gewerkschaften) und BDA (Arbeitgeber) die zentralen Interessenvertretungsinstanzen mit sozialpolitischem Auftrag, während auf Branchen- und Betriebsebene Einzelgewerkschaften und Branchenarbeitgeberverbände für die tarifpolitische Umsetzung der Interessen sorgen. Bis in die 1990er-Jahre gingen starke Verflechtungen zwischen Gewerkschaften und SPD mit mehrheitlich sozialpolitisch geprägten Mitgliedern des für Rentenreformen zentralen Sozialausschusses einher (Trampusch 2004a), was Ausdruck in konsensualen Politikmustern mit relativ geschlossenen 
Expertenzirkeln fand (Nullmeier u. Rüb 1993, S. 293-298). In den letzten beiden Jahrzehnten aber nahmen sowohl die tariflichen Machtressourcen als auch die sozialpartnerschaftlichen Verbindungen zu Parteien und Parlamenten ab (Wiß 2011). Zentrale Vetospieler für Rentenreformen sind die Regierungsparteien sowie, je nach Mehrheitsverhältnissen, die zweite Parlamentskammer (Bundesrat).

Eine Besonderheit Österreichs ist die verpflichtende Kammernmitgliedschaft von Arbeitgebern und Arbeitnehmern. Auf der Arbeiterseite vertreten die Arbeiterkammern zusammen mit stark zentralisierten Gewerkschaften (ÖGB) die Interessen der Beschäftigten, während diese Funktion auf der Kapitalseite den Wirtschaftskammern und der finanzstarken Industriellenvereinigung zukommt. Korporatistische Aushandlungsprozesse mit dem Ziel der Kompromissfindung präg $(\mathrm{t})$ en aufgrund zahlreicher parlamentarischer Gewerkschaftsmitglieder den politischen Prozess (Obinger u. Tálos 2006, S. 201). Der konsensuale Politikstil und die traditionelle starke sozialpartnerschaftliche Einflussnahme auf politische Entscheidungen haben seit den 1990er-Jahren nachgelassen (Schulze u. Schludi 2007). Ähnlich wie in Deutschland bilden die Regierungsparteien und die zweite Parlamentskammer die politischen Vetospieler.

Prozesse der Versäulung (Pillarisierung) und Fragmentierung haben in Belgien zu (sozialpartnerschaftlichen) Konflikten entlang von Regionen und Sprachen geführt. Einerseits sind Gewerkschaften und Arbeitgeberverbände auch wegen der Allgemeinverbindlichkeit der meisten Tarifverträge gut organisiert (sowie der gewerkschaftlichen Organisation der Arbeitslosenversicherung), andererseits aber auch fragmentiert (Anderson et al. 2007; Marier 2008), was einheitliche Positionen erschwert. Auf Seiten der Gewerkschaften steht der christliche Gewerkschaftsbund (CSC/ACV), welcher kooperativ-unterstützendend christdemokratischen Parteien verbunden ist, der radikaleren sozialistische Gewerkschaft (FGTB/ ABVV) gegenüber, die stärker auf Streiks als Druckmittel zurückgreift und mit den sozialistischen Parteien verknüpft ist (Marier 2008, S. 80). Auf der Arbeitgeberseite bildet der belgische Arbeitgeberverband FEB/VBO den größten Verbund. Mehr als Deutschland und Österreich ist Belgien aufgrund von Mehrparteienregierungen durch eine hohe Anzahl von Vetospielern gekennzeichnet (Anderson et al. 2007, S. 297; Natali 2008, S. 107).

Die fragmentierten italienischen Gewerkschaften zeichnen sich trotz eher geringer und fallender Organisationsgrade durch ihr hohes Mobilisierungspotenzial aus (Ferrera u. Jessoula 2007, S. 410-415). Die größten italienischen Gewerkschaften - die radikalere CGIL, die gemäßigtere CISL und die reformorientierte linke UIL - vertreten jeweils unterschiedliche politisch-ideologische Richtungen und haben Verbindungen zu verschiedenen Parteien. Wichtigster Arbeitgeberverband ist Confindustria, welche hauptsächlich Industrieunternehmen vertritt. Wie in Belgien sind Mehrparteienregierungen sowie die beiden Parlamentskammern verantwortlich für eine hohe Anzahl an Vetospielern.

Insgesamt stehen sich Deutschland und Österreich bezüglich der Form der Sozialpartnerschaft und Anzahl an Vetospielern näher. Beide Länder verfügen über umfassende Dachverbände und Zweiparteienregierungen. Einflussmöglichkeiten für Sozialpartner ergeben sich aus zum Teil engen Kontakten zu Parteien. Belgien und Italien dagegen bilden Bismarck-Länder mit stärker fragmentierten Sozial- 
partnern und Regierungen. Eine höhere Anzahl an Vetospielern resultiert aus Mehrparteienregierungen, was wiederum bei der Suche nach politischer Legitimität den Sozialpartner als Einfallstor für Verhandlungen zugutekommen kann.

\section{Die Sozialpartner im Politikgestaltungsprozess}

In den nächsten Abschnitten erfolgt die Analyse des sozialpartnerschaftlichen Einflusses im politischen Gesetzgebungsprozess in Deutschland, Österreich, Belgien und Italien, um so die de facto Vetomacht der Sozialpartner an politischen Vetopunkten aufzuzeigen. Hierbei wird bereits die formale Vetomacht, welche die Sozialpartner auf der kollektiven Ebene z.B. durch Tarifverträge besitzt, berücksichtigt, da diese wiederum auf deren de facto Vetomacht auf politischer Ebene zurückwirkt. Aus Gründen der analytischen Übersichtlichkeit und Vergleichbarkeit erfolgt die gesonderte Betrachtung der kollektiven Arena in Abschnitt 5. Entsprechend der theoretischen Überlegungen sollten Sozialpartner als Vetoakteure dann überwunden werden, wenn diese als Kompensation für Kürzungsmaßnahmen mit künftigen Machtgewinnen rechnen können, wozu sich die kollektive Ebene als Nebenschauplatz eignet. Gleichzeitig sollten die Sozialpartner bei nachlassendem Einfluss auf politischer Ebene ein erhöhtes Interesse an ihrem genuinen Feld - Tarifverhandlungen auf kollektiver Ebene - entwickeln.

\subsection{Deutschland: Auslaufmodell Konsens?}

Die letzte auf einem breiten parteipolitischen und sozialpartnerschaftlichen Konsens basierende Rentenreform war das 1989 verabschiedete Rentenreformgesetz 1992 (Schludi 2005; Busemeyer 2006), bevor sich dann die bürgerliche Regierung 1999 zusammen mit den Arbeitgebern gegen die Gewerkschaften und Opposition durchsetzte und stärkere soziale Einschnitte vornahm (Hassel u. Trampusch 2006; Schulze u. Jochem 2007, S. 683). Daraufhin verbündeten sich SPD und Gewerkschaften, um nach erfolgreicher Amtsübernahme 1998 durch eine rot-grüne Regierung wesentliche Bestandteile vorübergehend außer Kraft zu setzen. Das Anfangs etablierte Bündnis für Arbeit mit starker Einbindung der Sozialpartner scheiterte zwar aufgrund von innergewerkschaftlichen Divergenzen und Meinungsverschiedenheiten zwischen Regierung und Gewerkschaften über wegweisende Rentenreformen (Streeck 2003; Trampusch 2004b). Zugleich ermöglichte dies aber Teilallianzen der Regierung mit progressiven Gewerkschaften (IG BCE).

Weitreichende Rentenkürzungen ebenso wie die Einführung staatlich geförderter Zusatzrenten wurden 2001 durchgesetzt. Hauptsächlich am Ende des Gesetzgebungsprozess fanden die Interessen der Sozialpartner aufgrund deren Vetomacht bei der bAV Beachtung. Die Regierung baute auf eine breitere politische Legitimität, indem Zugeständnisse an die Gewerkschaften in Form eines Rechtsanspruches der Beschäftigten auf Entgeltumwandlung und Vorrang von Tarifverträgen bei der bAV erfolgten (Wiß 2011, S. 153-167). Die verabschiedete Reform war nach Ansicht der Regierung für eine nachhaltig finanzierbare Rente aber nicht ausreichend, weshalb 2004 das Rentenversicherungs-Nachhaltigkeitsgesetzes verabschiedet wurde und Kürzungen aufgrund der Einführung eines Nachhal- 
tigkeitsfaktors mit sich brachte. Unter Umgehung der Gewerkschaften und mit Hilfe zusätzlicher Legitimität durch eine Reform-Kommission konnte sich Kanzler Schröder erst durch ein Machtwort gegen Kritiker innerhalb der SPD durchsetzen (Schulze u. Jochem 2007, S. 693-695).

Bei der Organisationsreform der gesetzlichen Rentenversicherung 2004 konnte die Allianz aus Gewerkschaften und Arbeitgebern stärkere Eingriffsrechte des Staates verhindern, da diese ihre Rolle als formale Vetospieler bei der Implementierung ausspielen konnten (Klenk 2005; Wiß 2011, S. 173-176). Dieses Intermezzo klassenübergreifender Kooperation im Bereich der staatlichen Rente fand allerdings keine Forstsetzung, sobald es um inhaltliche Aspekte wie Finanzierung und Leistungshöhe ging. So erhöhte die Große Koalition 2007 mit Unterstützung durch Arbeitgeber und Forderungen der Rürup-Kommission die Altersgrenzen von 65 auf 67 Jahre, was den Gewerkschaften eine rentenpolitische Niederlage zufügte. In den letzten Jahren aber erfuhren gewerkschaftliche Interessen wieder eine stärkere Berücksichtigung (vgl. Wiß 2011, S. 178-183). Die befristete Sozialabgabenfreiheit bei Entgeltumwandlung in der bAV wurde 2007 auch auf Druck der Gewerkschaften entfristet, so dass die Sozialpartner ihr neu geschaffenes machtpolitisches Feld und tarifpolitische Kompetenzen festigen konnten.

In Deutschland führten die Rentenreformen seit den 1990er-Jahren zu einem Mehrsäulen-System, wobei die Sozialpartner eine Stärkung der betrieblichen Säule anstelle einer verpflichtenden privaten Säule durchsetzen konnten. Nachhaltige Reformen erfolgten unter Einbezug von de facto Vetospielern. Insgesamt aber mussten die Gewerkschaften im Bereich der staatlichen Renten im Gegensatz zum Arbeitgeberlager einen Einflussverlust hinnehmen.

\section{2 Österreich: Weitreichende Reformen durch Regierungswechsel}

Bis in die 1990er-Jahre standen in Österreich unter der Großen Koalition aus SPÖ und ÖVP korporatistische Aushandlungsprozesse im Vordergrund. Im Rahmen von mit Kürzungen verbundene Strukturreformen zur Erfüllung der Kriterien der Europäischen Wirtschafts- und Währungsunion wurden auf Druck der Gewerkschaften 1995 die Renten im öffentlichen Dienst verschont (Schulze u. Schludi 2007, S. 580-581). Vorschläge zur Einführung eines demografischen Faktors und weiterer Kürzungen 1997 stießen auf geteiltes Echo bei den Gewerkschaften (Schulze u. Schludi 2007, S. 584-587). Den Gewerkschaften gelang es, ihre Einwände durchzusetzen und die Regierung einigte sich mit den Sozialpartnern auf einen 15-jährigen Rentenberechnungszeitraum in allen Systemen und geringe Abschläge bei Frühverrentung (Obinger u. Tálos 2006, S. 87).

Das Jahr 2000 bildet eine Zäsur (critical juncture), indem die jahrzehntelang regierenden Volksparteien durch eine ÖVP/FPÖ-Regierung abgelöst wurde, welche einen neuen Politikstil unter Exklusion der Gewerkschaften pflegte (Tálos 2005). Wie bei früheren Reformprozessen reagierten die Gewerkschaften ablehnend auf Vorschläge zur Einführung eines Dreisäulen-Modells und höheren Abschlägen bei Frührenten. Ein Verhandlungsangebot von Seiten der Gewerkschaften sowie begleitende Demonstrationen konnten im Gegensatz zu früher die Verabschiedung des Gesetzes nicht verhindern. Mit Hilfe dieser Konfrontations- 
strategie konnte die Regierung das Rentenalter und die Abschläge bei Frühverrentung erhöhen (Schulze u. Schludi 2007, S. 587-589). Dagegen erfuhren sozialpartnerschaftliche Interessen auch unter der rechts-konservativen Regierung mehr Berücksichtigung, sobald die betriebliche Sozialpolitik in den Fokus rückte. Basierend auf einer gemeinsamen Vereinbarung der Sozialpartner wurde 2002 eine Stärkung der bAV mittels Expansion der Arbeitgeberleistung Abfertigung in Angriff genommen (Obinger u. Tálos 2006). Den Höhepunkt der Konfrontationen markierte der Reformprozess zum Budgetbegleitprozess 2003 (Obinger u. Tálos 2006, S. 89-90; Schulze u. Schludi 2007, S. 590-592). Trotz einer der größten Demonstrationen und Streiks in der Nachkriegsgeschichte Österreichs zeigte sich die Regierung unbeeindruckt und erweiterte den Rentenberechnungszeitraum von 15 auf 40 Jahre, um so einen Beitrag zur Haushaltssanierung zu leisten. Immerhin bewirkten die starken Proteste eine Deckelung der Verluste für Versicherte und Rentner auf zehn Prozent.

Dagegen wurden die Präsidenten der Sozialpartner durch ihre formale Vetomacht bei der Implementierung in der Selbstverwaltung 2004 in den Reformprozess zur Vereinheitlichung berufsspezifischer Rentensysteme und Einführung von individuellen Pensionskonten eingebunden (Tálos 2005, S. 285). Bei Reformen der bAV 2003 und 2005 waren die Sozialpartner ebenfalls eingebunden. Die Arbeitgeber konnten sich hier stärker durchsetzen, allerdings wurde auch eine sicherheitsorientierte Variante der bAV eingeführt.

Wie in Deutschland wurden Kürzungen der staatlichen Rente gegen den Widerstand der Gewerkschaften durchgesetzt, es gelang der Regierung aufgrund der starken Zentralisierung der Sozialpartner weniger, Teile der Gewerkschaften für Reformallianzen zu gewinnen. Die traditionelle korporatistische Verhandlungsdemokratie hat sich zwar vor allem nach dem Regierungswechsel 2000 abgenutzt (Tálos 2005, S. 294), bei Reformen betreffend die kollektive Ebene (Abfertigung, bAV) finden die Sozialpartner aber regelmäßig Gehör.

\subsection{Belgien: Fragmentierung als Einfallstor für Gewerkschaften}

Während die Gewerkschaften zu Beginn der 1980er-Jahre geplante Rentenkürzungen (Mainil-Reform) noch verhindert haben (Anderson et al. 2007, S. 323324; Marier 2008, S. 88), ermöglichte nachlassender Widerstand progressiver Teile der fragmentierten Gewerkschaften abgespeckte Reformen (Anderson et al. 2007, S. 325-326). Im Rahmen von tripartistischen Konsultationen konnte sich die Regierung im Rahmen einer Haushaltssanierung (St. Anna Plan) 1986 nicht mit den Sozialpartnern einigen, woraufhin sie sich mit einer geringfügigen Ausweitung des Rentenberechnungszeitraumes zufrieden gab. Ähnlich erging es der Regierung 1990, als Kürzungen der Beamtenpensionen trotz einer Reihe von Diskussionsrunden mit allen gesellschaftlichen Kräften nicht gegen die Haltung der Gewerkschaften durchgesetzt werden konnte. Im Jahr 1995 konnte der neue Sozialminister Colla zwar gegen den Widerstand beider Sozialpartner Verbesserungen in der Portabilität von Zusatzrenten durchsetzen, der Großteil der geplanten Reformen musste wegen gewerkschaftlicher Protester aber zurückgenommen werden (Anderson et al. 2007, S. 329-332). Nicht zuletzt aufgrund fehlender Kom- 
pensationsangebote z. B. auf kollektiver Ebene verweigerten die Sozialpartner ihre Zustimmung.

Vor dem Hintergrund des Beitritts zur Europäischen Wirtschafts- und Währungsunion sollten Rentenkürzungen 1995 die Neuverschuldung senken. Die Sozialpartner wurden zur Festlegung von Leitlinien eingeladen, wobei sich die Gewerkschaften erneut einem Reformpaket verweigerten (Anderson et al. 2007, S. 332-335). Als Reaktion beantragte die Regierung unter Umgehung des Parlaments Sonderbefugnisse in Form der Rahmengesetzgebung, um so Maßnahmen für ein finanziell nachhaltiges Sozialsystem zu erlassen. Mittels dieser Sonderbefugnis konnte die Regierung eine stärkere Steuerfinanzierung und Ausweitung der Referenzperiode für Frauen erlassen. Die Ex-Post-Legitimierung erfolgte durch eine spätere Zustimmung des Parlaments. Dagegen mündete eine gemeinsame Erklärung der Sozialpartner, basierend auf informellen Gesprächen mit der Regierung, in die Verabschiedung des Vandenbroucke-Gesetzes 2004. Die Sozialpartner stärkten die Tarifebene durch Einführung von verbindlichen branchenweiten Zusatzrenten (Anderson et al. 2007, S. 338). Zudem fand durch die Einführung von paritätischen Gremien für die bAV eine Stärkung der Mitbestimmungsrechte statt (De Deken 2011, S. 72).

Trotz Einschnitte gegen gewerkschaftlichen Widerstand basierten die meisten Reformen auf Verhandlungen mit den Sozialpartnern. Die Regierung konnte vor allem mit Hilfe der pragmatischeren christlichen Gewerkschaft kleinere Kürzungsmaßnahmen realisieren (Anderson et al. 2007, S. 340). Durch die starken Einflusskanäle der Gewerkschaften zu den Parlamentsparteien sind belgische Regierungen stärker auf eine Einigung mit den Sozialpartnern als mit oppositionellen Parteien angewiesen (Marier 2008, S. 105). Standen die Gewerkschaften der bAV bis Ende der 1990er-Jahre noch ablehnend gegenüber, so entwickelten sie im Laufe der Zeit ähnlich wie in Deutschland einen pragmatischeren Standpunkt, da anscheinend der organisatorische und machtpolitische Nutzen der bAV anerkannt wurde.

\subsection{Italien: Mobilisierung der Öffentlichkeit als Druckmittel}

Ein geringes wirtschaftliches Wachstum, demografische Verschiebungen und eine Währungs- und Finanzkrise machten in Italien zu Beginn der 1990er-Jahre Rentenreformen notwendig. Eine politisch-institutionelle Krise trug zunächst zu eher technokratisch geführten Regierungen bei, welche auch die Sozialpartner in Reformen einbezogen (Jessoula 2011, S. 160). So konnte das Kabinett unter Giuliano Amato 1992 durch informelle tripartistische Verhandlungen mit den Sozialpartnern eine Einigung über die Erhöhung des Rentenalters und Ausweitung des Rentenberechnungszeitraumes bei langen Übergangsphasen erzielen (Natali 2003). Die Einführung eines Regulierungsrahmens für Pensionsfonds 1993 legte den Grundstein für den Übergang zum Mehrsäulen-System. Um trotz hoher Sozialversicherungsbeiträge den Aufbau der bAV finanziell zu unterstützen, griff die Regierung auf das vom Arbeitgeber finanzierte Instrument Tfr zurück, um so ein Tor für Zusatzrenten zu schaffen (Ferrera u. Jessoula 2007, S. 431-433; Natali 2008, S. 121). Nur durch den Einbezug der Sozialpartner als formale Vetospieler 
auf betrieblicher Ebene konnte Tfr für die bAV nutzbar gemacht werden, wodurch sich die Gewerkschaften eine Demokratisierung des Kapitalismus und eigene Machtausweitung erhofften (Natali u. Rhodes 2008, S. 37).

Der neue Regierungschef Berlusconi gab 1994 landesweiten Demonstrationen und Streiks sowie Spannungen zwischen den Regierungsparteien nach und gab seine Pläne zu umfassenden Rentenkürzungen zugunsten marginaler Veränderungen (kurzzeitige Aussetzung der Frühverrentung) auf (Natali 2003, S. 27). Nicht zuletzt aufgrund dieses Scheiterns trat die Regierung Berlusconi kurz darauf zurück und wurde von der eher technokratischen Regierung unter Dini abgelöst. Hohe Interessenübereinkünfte und Verhandlungen zwischen Gewerkschaften und der Mitte-Links-Regierung (Natali 2003, S. 16) waren 1995 für Veränderungen auf kollektiver Ebene (bAV und Tfr) notwendig und dienten zugleich als Kompensation für die Umstellung der staatlichen Renten auf ein punkte- und beitragsorientiertes System zur Leistungsberechnung (Ferrera u. Jessoula 2007, S. 436-439; Jessoula 2011, S. 161). Jahrzehntelange Übergangsphasen verwässerten zudem die Schuldzuschreibung der Kürzungen (blame avoidance) (Natali 2008, S. 122).

Die zweite Berlusconi-Regierung scheiterte 2001 erneut mit einer Rentenreform - Ziel waren Veränderungen beim Tfr und Stärkung der dritten Säule - da er die Gewerkschaften nicht ausreichend eingebunden hatte, auf die er als zentrale Akteure der Implementierung aber angewiesen war (Ferrera u. Jessoula 2007, S. 443-444). Jahrelange Verhandlungen und Kontroversen mündeten schließlich unter Zustimmung der Sozialpartner in den Mechanismus der stillschweigenden Zustimmung (Jessoula 2011, S. 163), bei dem das Tfr für neue Beschäftigte bis auf Widerspruch automatisch in eine bAV umgewandelt wird.

Fragmentierte Regierungskoalitionen erforderten zusätzliche politische Legitimation, was der Interessendurchsetzung der mobilisierungsstarken Gewerkschaften zuträglich war. Auch ohne regelmäßige institutionelle Einbindung in den Gesetzgebungsprozess, wie dies stärker der Fall in Österreich, Belgien und Deutschland war, konnten sich die Gewerkschaften Einfluss auf Rentenreformen sichern. Ähnlich wie bei der Abfertigung in Österreich gelang der erst Schritt in Richtung Mehrsäulen-System über die Arbeitgeberzahlung Tfr, wodurch den Sozialpartner eine wichtige und vetomächtige Position im Gesamtsystem der Alterssicherung zukommt (Jessoula 2011, S. 156).

\subsection{Sozialpartner als de facto Vetospieler an formalen Vetopunkten}

Rentenreformen in Ländern mit mehreren Blockademöglichkeiten sind möglich, wenn Gewerkschaften im Gegenzug zu Kürzungen in der staatlichen Rente neue Handlungsspielräume in der bAV in Aussicht gestellt werden. Die Länderbeispiele zeigen, dass Regierungen in Bismarck-Ländern beim Übergang zu MehrsäulenSystemen mehrheitlich auf den Einbezug der Gewerkschaften gesetzt haben, einerseits um die Legitimität dieses Schrittes zu erhöhen, andererseits aufgrund deren Vetomacht bei der Umsetzung der Reformen und innerhalb der bAV auf kollektiver Ebene. Zentrale Bedingungen zur Überwindung gewerkschaftlichen Widerstandes waren Reformallianzen zumindest mit Teilen des Gewerkschaftslagers bei gleichzeitigen Zugeständnissen auf kollektiver Ebene in Form von höhe- 
rer Verantwortung und Machtgewinn in der bAV. Nachlassender Einfluss der Gewerkschaften v.a. in Deutschland und Österreich zu Beginn der 2000er könnte deren Kompromissbereitschaft erhöht und die Rückverlagerung der Gestaltungsmacht auf die kollektive Ebene verstärkt haben. Allerdings gelang es einigen Regierungen mittels Konfrontation und Machtfragen, Kürzungen teilweise gegen den Widerstand der Gewerkschaften durchzusetzen. Der Einbezug von Gewerkschaften findet gegenüber der Vergangenheit insgesamt weniger kontinuierlich nun vermehrt punktuell statt und fast automatisch, wenn Reformen direkt oder indirekt die kollektive Ebene zum Inhalt haben.

\section{Die Umsetzung der Reformen und Selbstregulierung der betrieblichen Altersvorsorge}

Die Analyse der kollektiven Arena verdeutlicht die neuen und bereits in der Vergangenheit potenziell bestehenden Handlungsspielräume der Sozialpartner inklusive deren Stellenwert für die Sozialpartner einerseits und für das System der Alterssicherung andererseits. Nur so kann die gesamte Vetomacht der Sozialpartner bestimmt und Verbindungslinien zwischen politischer und kollektiver Ebene sowie zwischen Politikgestaltung und Implementierung verdeutlicht werden. Welchen Einfluss haben die Sozialpartner auf die Implementierung und welche Bedeutung kommt der bAV zu? Aufgrund nachlassenden Einflusses auf die politische Arena und im Zuge von Reformpaketen sollte die bAV für die Sozialpartner wichtiger werden, um so durch eine stärkere formale Vetomacht auf kollektiver Ebene auch wieder mehr Einfluss auf politischer Ebene zu erlangen. Gleichzeitig erhöht der Einbezug der sozialpartnerschaftlichen Ebene die Kompensationschancen für Regierungen. Um die Gemeinsamkeiten und Unterschiede in den einzelnen Ländern hervorzuheben und besser vergleichbar zu machen, werden die Charakteristika der bAV in den einzelnen Ländern im Folgenden entlang von thematischen Einheiten dargestellt.

\subsection{Formen und Verbreitung der bAV}

Die Verbreitung der bAV, die in allen Ländern weitestgehend freiwillig ist und den Sozialpartnern Spielräume lässt, hat seit Ende der 1990er-Jahre stark zugenommen. Die Abdeckung von Beschäftigten mit einer bAV ist heute in Deutschland und Belgien mit 60-70 Prozent höher, Österreich und Italien weisen mit knapp 32 Prozent niedrigere Raten bei gegenüber Deutschland und Belgien höheren staatlichen Renten auf.

Während in Deutschland in der Vergangenheit die bAV auf Firmenebene und für qualifizierte männliche Beschäftigte dominierte, haben die vergangenen Reformen durch den Rechtsanspruch auf Entgeltumwandlung und den Tarifvorbehalt die Rolle der Sozialpartner gestärkt, so dass die bAV seit 2001 Bestandteil in hunderten von Tarifverträgen ist. Im Vergleich zu 14 Millionen Versicherten vor den Reformen von 2001 verfügen inzwischen 17-19 Millionen Beschäftigte über eine bAV (ca. 65-70 Prozent der sozialversicherungspflichtig Beschäftigten) (Wiß 2011, S. 194-195). Tarifliches Entgelt darf nur per gesonderten Tarifvertrag in eine bAV 
umgewandelt werden, was den Sozialpartnern zu neuer tarifpolitischer Macht verhilft. Auch in Belgien ist die Bedeutung der bAV seit 2000 kontinuierlich angestiegen, so verfügten im Jahr 200759 Prozent aller Beschäftigten über eine bAV, gegenüber noch 44 Prozent im Jahr 2000. Vor allem die branchenweite und mitbestimmte bAV auf Initiative der Sozialpartner hat in den letzten Jahren kontinuierlich zugenommen (De Deken 2011, S. 73-79). Im Fall der verpflichtenden Arbeitgeberleistung Abfertigung haben Arbeitnehmer in Österreich bei Beendigung des Dienstverhältnisses einen Anspruch auf Leistungen, wobei neben der Einzahlung in betriebliche Vorsorgekassen auch andere Verwendungsmöglichkeiten bestehen. Aufgrund von Neuerungen werden inzwischen 60-80 Prozent aller Beschäftigten erreicht werden, gegenüber 15 Prozent vor den Reformen (Obinger u. Tálos 2006, S. 91). Auch in der freiwilligen Pensionskasse können die Sozialpartner Erfolge verzeichnen, im Jahr 2010 verfügten zusätzlich 700.000 Versicherte über eine bAV (ca. 20 Prozent der abhängig Beschäftigten), gegenüber nur 77.0001995 (2,5 Prozent). Ähnlich der Abfertigung in Österreich existiert auch in Italien eine verpflichtende Arbeitgeberleistung (Tfr), die nicht zwangsläufig für die Altersvorsorge genutzt werden muss, allerdings seit den Reformen zunächst automatisch in eine bAV überführt wird (Jessoula 2011, S. 165). Anfang der 1990er-Jahre besaßen noch weniger als fünf Prozent der Beschäftigten eine bAV (Ferrera u. Jessoula 2007, S. 442), dank der jüngsten Reformen und Anstrengungen der Sozialpartner sind es heute knapp 32 Prozent. Insgesamt hat sich die bAV in allen Ländern seit den Reformen positiv entwickelt, den Sozialpartnern scheint im Rahmen der Reformprozesse eine machtpolitische Ausweitung auf kollektiver Ebene gelungen zu sein, ansteigende bAV-Anwartschaften übertragen ihnen höhere Verantwortung.

\subsection{Sozialpartnerschaftliche Beteiligungsformen}

Neben dem quantitativen Bedeutungsgewinn der bAV gibt die Beteiligung der Gewerkschaften und Arbeitgeber bei Abschluss und Durchführung von betrieblichen Rentenplänen Aufschluss über deren formale Vetomacht auf kollektiver Ebene.

Die in der Vergangenheit dominierende bAV auf Firmenebene in Deutschland räumte den Betriebsräten je nach Unternehmen nur eingeschränkte Mitspracherechte ein (OECD 2008, S. 226-227; Ebbinghaus u. Wiß 2011, S. 21). Demgegenüber basieren die seit 2001 neu etablierten kollektiven Branchenversorgungswerken auf gemeinsamen Initiativen und Tarifverträgen von Gewerkschaften und Arbeitgeberverbänden. In Institutionen wie MetallRente, ChemieVersorgungswerk, den Sozialkassen im Baugewerbe und der Versorgungsanstalt des Bundes und der Länder (VBL) im öffentlichen Dienst sind die Sozialpartner paritätisch in verschiedenen Ausschüssen vertreten, was ihnen Einfluss z.B. auf Entscheidungen über Anlagestrategien und Auswahl der mit der Durchführung beauftragten Finanzinstitute sichert. Die unternehmensbasierte bAV in Belgien unterliegt grundsätzlich der Mitsprache der Betriebsräte (De Deken 2011, S. 83-84), allerdings entfällt dies bei alleinig vom Arbeitgeber finanzierten bAV-Verträgen ohne solidarische Elemente. Demgegenüber wurde die Mitbestimmung durch das Vandenbroucke Gesetz 2003 - die Sozialpartner waren maßgeblich beteiligt - gestärkt, welches eine paritätische Kontrolle von branchenweiten Betriebsrenten und fir- 
menspezifischer Altersvorsorge mit solidarischen Elementen vorsieht. In Österreich erfolgt die Auslagerung der Abfertigung ebenso wie Einzahlungen in Pensionskassen auf der Grundlage von Betriebsvereinbarungen bzw. Tarifverträgen. Kapitalvertreter verfügen in den Aufsichtsräten über die Mehrheit (OECD 2008, S. 203). Paritätisch zusammengesetzt ist dagegen ein beratendes Gremium bei Pensionskassen und betrieblichen Kollektivversicherungen, das allerdings nur über Informationsrechte besitzt. Neben dem $T f r$, das Sozialpartnerverhandlungen unterliegt, basieren in Italien geschlossene Pensionsfonds auf Tarifverträgen, zusätzlich bestehen Mitspracherechte über den paritätisch besetzten Vorstand. Die Governance-Struktur von offenen Pensionsfonds ist dagegen schlanker, Arbeitnehmer verfügen weder im Vorstand noch im Aufsichtsrat über Mitspracherechte (Jessoula 2011, S. 174-175).

Neben der quantitativen Ausweitung der Verantwortung von Sozialpartnern für die Alterssicherung aufgrund steigender bAV-Anwartschaften macht sich der Machtgewinn auch qualitativ bemerkbar. Zum einen ist die bAV immer häufiger Bestandteil von Tarifverträgen und damit auch der Mitbestimmung, zum anderen partizipieren die Sozialpartner direkt oder indirekt am Aufbau, der Organisation und Durchführung von Pensionseinrichtungen.

\subsection{Finanzierung}

Durch den Blick auf die Finanzierungsseite der bAV können Schlüsse bezüglich der finanziellen Macht gezogen werden. Wer finanziert verfügt über Entscheidungsbefugnisse und Einfluss und ist damit bei Reformen zu berücksichtigen. Zusätzlich können im Zuge von gegenläufigen Entwicklungen auf politischer und kollektiver Ebene z. B. staatliche Rentenkürzungen mit Steuerbefreiungen oder sonstigen Vergünstigungen in der bAV kompensiert werden. Je nachdem, ob Arbeitgeber oder Arbeitnehmer einzahlen, hat zudem Einfluss auf Tarifverhandlungen und damit die Vetomacht der Sozialpartner im Bereich der bAV.

Während die traditionellen auf Buchungsrückstellungen basierenden Durchführungswege in Deutschland alleinig vom Arbeitgeber finanziert wurden, werden seit 2002 Beiträge zu kapitalfundierten Durchführungswegen paritätisch oder alleinig vom Arbeitnehmer geleistet, wodurch Kollektivverhandlungen aufgrund des Tarifvorbehaltes notwendig sind. Bei der steuer- und abgabenfreien Entgeltumwandlung werden durchschnittlich ca. 1200 Euro pro Jahr in eine bAV eingezahlt. Belgien weist im europäischen Vergleich eine der geringsten Beitragszahlungen zur bAV auf, mit höheren Beiträgen zu individuellen Rentensparplänen. In der branchenweiten bAV variiert die Arbeitgeberfinanzierung zwischen 0,5 und 4,2 Prozent der Bruttolöhne, nur selten beteiligen sich auch die Arbeitnehmer. Die niedrigen Arbeitgeberbeiträge können teilweise auf eine von der Regierung unter Beteiligung eines tripartistischen Gremiums erlassene Obergrenze von Lohnzuwächsen zurückgeführt werden, an die auch Tarifverhandlungen zur bAV (mit Ausnahme der solidarischen bAV) gebunden sind (De Deken 2011, S. 79). In Österreich zahlt der Arbeitgeber zur Abfertigung einen monatlichen Pflichtbeitrag in Höhe von 1,53 Prozent des Bruttogehalts. Bei der freiwilligen bAV sind Arbeitgeberbeiträge obligatorisch, individualisierte Formen wie die nur vom Arbeitnehmer 
finanzierte Entgeltumwandlung in Deutschland sind nicht möglich. Die obligatorischen Beiträge der Arbeitgeber zum Tfr in Italien betragen 6,91 Prozent des Bruttogehalts, wesentlich mehr als beim österreichischen Pendant. Zur durchschnittlichen Finanzierung der geschlossenen Pensionsfonds in Höhe von 9,29 Prozent des Gehalts tragen zusätzlich zum Tfr Arbeitgeber- und Arbeitnehmerbeiträge bei (Jessoula 2011, S. 172-173). Abgesehen von den Sonderformen Tfr in Italien und Abfertigung in Österreich dominieren Mischfinanzierungen bei steigenden Arbeitnehmeranteilen die bAV-Landschaft, so dass hier sowohl Gewerkschaften als auch Arbeitgeber an wichtigen Schaltstellen der bAV sitzen, was Verhandlungen notwendig macht und Einfluss sichert. Seit den 1990er-Jahren sind vielfältige Steuervergünstigungen für Beiträge zur bAV in allen Ländern verabschiedet worden, um den Ausbau der bAV bei sinkenden staatlichen Renten zu fördern.

\subsection{Pensionsfondskapitalismus}

Die Höhe der Pensionsfondsanlagen und damit auch von kollektiv vereinbarten Systemen der bAV hat Auswirkungen auf die Finanzmärkte (und umgekehrt). Dies ermöglicht Gewerkschaften (theoretisch) durch ihre zentrale Rolle bei der kollektiven bAV eine „Zähmung“ des Kapitalismus, indem durch gewerkschaftliche Beteiligung Einfluss auf Investitionen und Unternehmen und damit auf Finanzmärkte insgesamt genommen werden kann. Im Pensionsfonds der deutschen Metallrente werden z. B. Investitionen auf Druck der Gewerkschaften vorrangig in nachhaltige und ethische Investments vorgenommen.

Die bis in die 1990er-Jahre dominierenden unternehmensinternen Buchungsrückstellungen in Deutschland sind auch heute noch für rund die Hälfte aller Deckungsmittel der bAV verantwortlich, wobei kapitalfundierte Durchführungswege auf dem Vormarsch sind. In Österreich basierte das alte System der Abfertigung ebenso wie das alte Tfr-System in Italien auf innerbetrieblicher Finanzierung, so dass die Beiträge den Finanzmärkten nicht zur Verfügung standen. Seit deren Reformierung fließen die Beiträge nun in externe kapitalfundierte Formen. Der Anteil von Pensionsfonds gemessen am Bruttoinlandsprodukt war 2010 in Österreich und Deutschland am höchsten (siehe Tabelle 3). Den stärksten Anstieg konnte Italien mit einer durchschnittlichen jährlichen Wachstumsrate von 15,8 Prozent verzeichnen. Der gegenüber den anderen Ländern nur schwache Anstieg in Belgien ist auf starke Einbrüche in Folge der Finanzmarktkrisen von 2002 und 2008 zurückzuführen. Eine steigende Bedeutung der kapitalgedeckten Pensionsfonds - und damit auch der Rolle der Sozialpartner - wird zudem deutlich, wenn die heutigen Beiträge die Leistungen übertreffen. 
Tabelle 3: Pensionsfondskapitalismus 2001-2010

\begin{tabular}{lccccc}
\hline & $\begin{array}{c}\text { Investitionen } \\
\text { von } \\
\begin{array}{c}\text { Pensionsfonds } \\
\text { in \% BIP } \\
2010\end{array}\end{array}$ & $\begin{array}{c}\text { Pensionsfondsanlagen } \\
\text { in Mrd. US Dollar }\end{array}$ & $\begin{array}{c}\text { Beiträge } \\
\text { in \% BIP }\end{array}$ & $\begin{array}{c}\text { Leistungen } \\
\text { in \% BIP }\end{array}$ \\
\hline Österreich & 5,3 & 19,8 & $+14,9 \%$ & 0,4 & 2010 \\
Deutschland & 5,2 & 171,4 & $+11,4 \%$ & 0,5 & 0,2 \\
Italien & 4,6 & 93,8 & $+15,8 \%$ & 0,6 & 0,2 \\
Belgien & 3,8 & 17,6 & $+3,6 \%$ & 0,4 & 0,2 \\
\hline
\end{tabular}

Anmerkungen: Nur kapitalfundierte System werden berücksichtigt; Deutschland ohne Direktversicherungen. Quellen: OECD Global Pension Statistics; OECD 2011b, S. 9.

\subsection{Die Sozialpartner als formale Vetospieler an kollektiven Vetopunkten}

In allen hier untersuchten Ländern ist der klare Trend ansteigender bAV-Anwartschaften seit Ende der 1990er-Jahre zu konstatieren, wobei externe kapitalfundierte Formen unter Mitsprache der Sozialpartner über Tarifverträge aufgrund von Reformen an Bedeutung gewinnen. Quantitativ und qualitativ ging der Ausbau der bAV mit mehr Mitsprache der Sozialpartner einher und ist damit eine zentrale Bedingung, um Gewerkschaften für Reformpakete zu gewinnen. Österreich und Italien hinken bezüglich der Abdeckungsrate von Versicherten Deutschland und Belgien hinterher, wobei die obligatorischen Arbeitgeberleistungen in Form der Abfertigung (Österreich) und Tfr (Italien) berücksichtigt werden müssen, die nicht immer eindeutig zur bAV gezählt werden können. Entsprechend den kooperativen industriellen Beziehungen basieren die neuen Formen der bAV vor allem in Deutschland, Belgien und Italien auf branchenweiten Vereinbarungen, während in Österreich Firmenvereinbarungen überwiegen aber auch branchenweite Tarifverträge auf dem Vormarsch sind. Insgesamt konnten die Sozialpartner erfolgreich ihre Beteiligung am Ausbau der Zusatzrenten sichern und als Kompensation für Kürzungen der staatlichen Rente neue machtpolitische Felder besetzen. Ihre Rolle als Vetospieler in der kollektiven Arena bei der Implementierung und Selbstregulierung erfuhr eine Aufwertung, was auf der staatlichen Ebene in Reformprozessen entsprechende Berücksichtigung fand.

\section{Resümee: Rentenreformen trotz multipler Vetomöglichkeiten}

Ziel der vorangegangen Analyse war zu klären, wieso Rentenreformen in Bismarck-Ländern möglich sind und welchen Einfluss die Sozialpartner hierbei im politischen Gesetzgebungs- und Implementierungsprozess haben. Da die klassische Ansätze zu Vetomöglichkeiten Reformaktivitäten in Ländern mit zahlreichen Vetoakteuren nicht ausreichend erklären können, wurden diese durch stärkere Berücksichtigung der Sozialpartner dennoch nutzbar gemacht. Ergänzungen der Ansätze zu Vetospielern und Vetopunkten ermöglichen a) die Berücksichtigung von staatli- 
chen und nicht-staatlichen Akteuren, b) die Integration von politischer (Politikgestaltung) und kollektiver Ebene (Implementation/Selbstregulierung) und weisen damit c) auf die gesamte Vetomacht einzelner Akteure inklusive der Verbindungslinien zwischen politischer und kollektiver Arena hin. Der Einbezug potenzieller Machtarenen abseits der politischen Bühne, wie im Falle der bAV, statt der Fokussierung nur auf den parlamentarischen-politischen Raum und staatliche Politik leistet einen Beitrag zur Erklärung von Reformaktivitäten trotz multipler Vetomöglichkeiten. Der Einbezug von de facto Vetoakteuren und deren Bedeutung auf nicht-staatlicher - hier kollektiver - Ebene hat Raum für zum Teil einschneidende Rentenreformen in Bismarck-Ländern eröffnet. Bisherige Argumente zu Reformpakten lediglich auf staatlicher Ebene reichen nicht aus, da die Sozialpartner dort nur wenig hinzugewinnen können. Die teils de facto teils formalen Vetospieler Sozialpartner können dagegen für Reformen gewonnen werden, wenn im Gegenzug ein Machtgewinn durch den Ausbau der bAV in Aussicht gestellt wird. Politische und kollektive Vetopunkte müssen zueinander in Verbindung gesetzt werden, zunächst vermutete Blockaden an politischen Schaltstellen (die theoretisch zu weniger Reformen führen sollten) können durch Pakete/Kompensationen bei der Implementierung und Selbstregulierung umgangen bzw. überwunden werden. Viele Vetopunkte erhöhen zudem die Anzahl potenzieller Allianzen (z. B. konnten Differenzen innerhalb der Gewerkschaften ausgenutzt werden, indem Reformallianzen mit progressiven Gewerkschaften geschlossen wurden). Gleichzeitig erhöhen sich auch die politische Legitimität und damit die Verantwortung für Reforminhalte, was hilfreich für die Nichtbestrafung bei künftigen Wahlen ist. Generell ist die $\mathrm{Zu}-$ sammenarbeit mit Sozialpartnern aufgrund deren begrenzter de facto Vetomacht keine Voraussetzung für Reformen der staatlichen Renten. Allerdings erfahren Gewerkschaften und Arbeitgeberverbände eine stärkere Berücksichtigung beim Übergang zu Mehrsäulen-Systemen, da die Ausbreitung ebenso wie Detailregelungen der bAV unmittelbar von Verhandlungen der Sozialpartner abhängen. Die Kombination von Rentenkürzungen mit neuen machtpolitischen Feldern für die Sozialpartner scheint somit ein wesentlicher Faktor für die höheren Reformaktivitäten in Bismarck-Ländern zu sein. Eine Ausweitung des hier entwickelten analytischen Rahmens auf weitere Politikfelder bietet sich für nachfolgende Forschungsarbeiten zu Reformprozessen in Ländern mit vielen Vetospielern an.

Welchen Einfluss konnten die Sozialpartner auf den politischen Gesetzgebungsund Implementierungsprozess nehmen? Gemeinsam ist allen hier untersuchten Ländern die sozialpartnerschaftliche Flankierung des Wandels vom Einsäulenzum Mehrsäulen-System als koordinierter Privatisierungspfad konservativer Wohlfahrtsstaaten. Sowohl der Reformprozess als auch die Implementierung und Selbstregulierung folgen weitestgehend ähnlichen Mustern. Erfolgreiche Rentenreformen sind vor allem dann auszumachen, wenn die Sozialpartner bei Kürzungsmaßnahmen eingebunden werden und gleichzeitig Paketlösungen durch Ausbau der kollektiven bAV erreicht werden (vgl. die Reformen in Deutschland 2001 und 2007, in Österreich 2002 und 2004, in Belgien 2003 und in Italien 1995 und 2007). Die Einbindung von Sozialpartnern als de facto Vetospieler bei Reformen nur der staatlichen Rente hängt stark vom Regierungsstil und dem Willen zur Berücksichtigung nicht-staatlicher Akteure ab. Bei Reformen der bAV fin- 
den Sozialpartner dagegen fast automatisch Berücksichtigung. Konfrontative Strategien führten dagegen in einigen Fällen zum Scheitern (so die Reform in Deutschland 1997, in Belgien Anfang 1995 und in Italien 1994). Allerdings finden sich auch (wenige) Reformprozesse, die Kürzungsmaßnahmen gegen den Widerstand der Gewerkschaften durchsetzten (so die Reform in Belgien Ende 1995, in Österreich 2000), wenn Regierungen konfrontationsbereit sind und den Sozialpartnern aufgrund fehlender formaler Vetomacht bei der Implementierung auch de facto Vetomacht auf staatlicher Ebene fehlt.

Als Ergebnis wurden insgesamt künftige Leistungen aus umlagefinanzierten staatlichen Rentensystemen gekürzt und Zusatzrenten forciert. Zudem unterlag auch die Form der bAV einem Änderungsprozess. Intern finanzierte Durchführungswege mit Leistungszusagen werden zurückgefahren, im Gegenzug findet eine Stärkung von externen kapitalfundierten Durchführungswegen mit Beitragszusage statt. Dennoch folgt dieser Pfad keiner reinen Marktstrategie, sondern vielmehr einer Art kollektiver Dezentralisierung in dem Sinne, dass der Ausbau in Form von Tarifverträgen und kollektiven Versorgungswerken, also unter Beteiligung von Gewerkschaften und Arbeitgeberverbänden erfolgt - branchenweite Tarifverträge und kollektive Versorgungswerke in Deutschland, branchenweite Systeme mit erga omnes Wirkung in Belgien, überbetriebliche Pensionskassen und Umwandlung der Abfertigung in Österreich sowie geschlossene Pensionsfonds und $T$ fr-Einzahlung in Italien.

Bei der Neugestaltung des Alterssicherungssystems wurde auf koordinierende Institutionen wie Sozialpartnerschaft zurückgegriffen, so dass die bAV als Subsphäre in das entsprechende gesamtgesellschaftliche System eingebettet wurde. Zwar sprechen die Kürzung der staatlichen Rente und die teilweise Verlagerung der Altersvorsorge in den nicht-staatlichen Bereich zunächst gegen ein traditionelles konservatives Wohlfahrtsregime und eine koordinierte Marktökonomie, allerdings findet die Organisation und Durchführung der bAV mittels klassenübergreifender Kooperation statt, was wiederum zentrales Charakteristikum von Koordinierung ist. Auch in Mehrsäulen-Systemen sind die Unterschiede zwischen kontinentalen und liberalen Marktökonomien größer, als die hier konstatierten Unterschiede innerhalb der Bismarck-Gruppe. Für eine empirische Untermauerung bietet sich für künftige Studien eine systematische Gegenüberstellung der hier erzielten Ergebnisse mit Privatisierungspfaden in Ländern, die nicht zur Bismarck-Fraktion zählen, an. 


\section{Literatur}

Anderson, Karen M., Sanneke Kuipers, Isabelle Schulze und Wendy van den Nouland. 2007. Belgium. Linguistic Veto Players and Pension Reform. In The Handbook of West European Pension Politics, Hrsg. Ellen M. Immergut, Karen M. Anderson und Isabelle Schulze, 297-346. Oxford: Oxford University Press.

Béland, Daniel. 2001. Does Labor Matter? Institutions, labor unions and pension reform in France and the United States. Journal of Public Policy 21:153-172.

Bonoli, Giuliano. 2000. The Politics of Pension Reform. Institutions and Policy Change in Western Europe. Cambridge: Cambridge University Press.

Bonoli, Giuliano. 2001. Political Institutions, Veto Points, and the Process of Welfare State Adaptation. In The New Politics of the Welfare State, Hrsg. Paul Pierson, 238-264. New York: Oxford University Press.

Busemeyer, Marius R. 2006. Moving the Unmovable. Political Strategies of Pension Reform in Germany. German Policy Studies 3:400-445.

Collier, David .1993. The Comparative Method. In Political Science. The State of the Discipline II, Hrsg. Ada W. Finifter, 105-119. Washington: American Political Science Association.

De Deken, Johan J. 2011. Belgium. The Paradox of Persisting Voluntarism in a Corporatist Welfare State. In The Varieties of Pension Governance. Pension Privatization in Europe, Hrsg. Bernhard Ebbinghaus, 57-88. Oxford: Oxford University Press.

Ebbinghaus, Bernhard (Hrsg.). 2011. The Varieties of Pension Governance. Pension Privatization in Europe. Oxford: Oxford University Press.

Ebbinghaus, Bernhard, und Tobias Wiß. 2011. Taming pension fund capitalism in Europe. collective and state regulation in times of crisis. Transfer - European Review of Labour and Research 17:15-28.

Esping-Andersen, Gøsta (Hrsg.). 1996. Welfare States in Transition. National Adaptations in Global Economies. London: Sage.

Ferrera, Maurizio, und Matteo Jessoula. 2007. Italy. A Narrow Gate for Path-shift. In The Handbook of West European Pension Politics, Hrsg. Karen M. Anderson, Ellen M. Immergut und Isabelle Schulze, 396-453. Oxford: Oxford University Press.

Hacker, Jacob S. 2005. Policy Drift. The Hidden Politics of US Welfare State Retrenchment. In Beyond Continuity. Institutional Change in Advanced Political Economies, Hrsg. Wolfgang Streeck und Kathleen Thelen, 40-82. Oxford: Oxford University Press.

Hall, Peter A., und David Soskice (Hrsg.). 2001. Varieties of Capitalism. New York: Oxford University Press.

Hassel, Anke, und Christine Trampusch. 2006. Verbände und Parteien. Die Dynamik von Parteikonflikten und die Erosion des Korporatismus. In Transformationen des Kapitalismus. Festschrift für Wolfgang Streeck zum sechzigsten Geburtstag, Hrsg. Jens Beckert, Bernhard Ebbinghaus, Anke Hassel und Philip Manow, 111-132. Frankfurt a.M.: Campus Verlag.

Häusermann, Silja. 2010. The Politics of Welfare State Reform in Continental Europe. Modernization in Hard Times. New York: Cambridge University Press.

Héritier, Adrienne. 2001. Differential Europe. National Administrative Responses to Community Policy. In Transforming Europe. Europeanization and Domestic Change, Hrsg. 
Maria Green Cowles, James Caporaso und Thomas Risse, 44-59. Ithaca: Cornell University Press.

Huber, Evelyne, und John D. Stephens. 2001. Development and Crisis of the Welfare State. Parties and Policies in Global Markets. Chicago: Chicago University Press.

Immergut, Ellen M. 1992. Health Politics. Interests and Institutions in Western Europe. Cambridge u. a.: Cambridge University Press.

Immergut, Ellen M., und Tarik Abou-Chadi. 2010. Political and Institutional Determinants of Social Legislation. The Role of Veto Points, Veto Players, and Electoral Pressure. BGSS Working Paper No. 3. Berlin: Department of Social Sciences, Humboldt-Universität zu Berlin.

Jessoula, Matteo. 2011. Italy. From Bismarckian Pensions to Multipillarization under Adverse Conditions. In The Varieties of Pension Governance. Pension Privatization in Europe, Hrsg. Bernhard Ebbinghaus, 151-179. Oxford: Oxford University Press.

Kaiser, André. 1998. Vetopunkte der Demokratie. Eine Kritik neuerer Ansätze der Demokratietypologie und ein Alternativvorschlag. Zeitschrift für Parlamentsfragen 98:525-541.

Kitschelt, Herbert. 2001. Partisan Competition and Welfare State Retrenchment. When Do Politicians Choose Unpopular Policies? In The New Politics of the Welfare State, Hrsg. Paul Pierson, 265-302. New York: Oxford University Press.

Klenk, Tanja. 2005. Die Organisationsreform in der gesetzlichen Rentenversicherung. Eine Betrachtung aus politikwissenschaftlicher Perspektive. Zeitschrift für Sozialreform 51:94-127.

Lijphart, Arend. 1999. Patterns of Democracy. Government Forms and Performance in Thirty-Six Countries. New Haven/London: Yale University Press.

Lindvall, Johannes. 2010. Power Sharing and Reform Capacity. Journal of Theoretical Politics 22:359-376.

Mahoney, James. 2003. Strategies of Casual Assessment in Comparative Historical Analysis. In Comparative Historical Analysis in the Social Sciences, Hrsg. James Mahoney und Dietrich Rueschemeyer, 337-372. Cambridge: Cambridge University Press.

Mares, Isabela. 2004. Warum die Wirtschaft den Sozialstaat braucht. Ein historischer Ländervergleich. Frankfurt a.M./ New York: Campus Verlag.

Marier, Patrik. 2008. Pension Politics. Consensus and social conflict in ageing societies. London/New York: Routledge.

Myles, John, und Paul Pierson. 2001. The Comparative Political Economy of Pension Reform. In The New Politics of the Welfare State, Hrsg. Paul Pierson, 305-333. New York: Oxford University Press.

Natali, David. 2003. The Role of Trade Unions in the Pension Reforms in France and Italy. EUI Working Paper SPS No. 2003/3. Florenz: European University Institute.

Natali, David. 2008. Pensions in Europe, European Pensions. The Evolution of Pension Policy at National and Supranational Level. Brüssel: Peter Lang.

Natali, David, und Martin Rhodes. 2008. The ,new politics' of pension reforms in Continental Europe. In Pension Reform in Europe. Politics, policies and outcomes, Hrsg. Camila Arza und Martin Kohli, 25-46. London/New York: Routledge.

Nullmeier, Frank, und Friedbert W. Rüb. 1993. Die Transformation der Sozialpolitik. Frankfurt a.M./New York: Campus Verlag. 
Obinger, Herbert, und Emmerich Tálos. 2006. Sozialstaat Österreich zwischen Kontinuität und Umbau. Eine Bilanz der ÖVP/FPÖ/BZÖ-Koalition. Wiesbaden: VS Verlag für Sozialwissenschaften.

OECD. 2008. Complementary and Private Pensions throughout the World 2008. Paris: OECD.

OECD. 2011a. Pensions at a Glance 2011. Retirement-Income Systems in OECD and G20 Countries. Paris: OECD.

OECD. 2011b. Pension Markets in Focus July 2011(8). Paris: OECD.

Orenstein, Mitchell A. 2008. Privatizing Pensions. The Transnational Campaign for Social Security Reforms. Princeton: Princeton University Press.

Palier, Bruno (Hrsg.). 2010. A long goodbye to Bismarck? The politics of welfare reforms in continental Europe. Amsterdam: Amsterdam University Press.

Pierson, Paul. 1996. The New Politics of the Welfare State. World Politics 48:143-179.

Pierson, Paul. 1998. Irresistible Forces, Immovable Objects. Post-Industrial Welfare States Confront Permanent Austerity. Journal of European Public Policy 5:539-560.

Schludi, Martin. 2005. The Reform of Bismarckian Pension Systems. A Comparison of Pension Politics in Austria, France, Germany, Italy and Sweden. Amsterdam: Amsterdam University Press.

Schulze, Isabelle, und Sven Jochem. 2007. Germany. Beyond Policy Gridlock. In The Handbook of West European Pension Politics, Hrsg. Ellen M. Immergut, Karen M. Anderson und Isabelle Schulze, 660-710. Oxford: Oxford University Press.

Schulze, Isabelle, und Martin Schludi. 2007. Austria. From Electoral Cartels to Competitive Coalition-building. In The Handbook of West European Pension Politics, Hrsg. Ellen M. Immergut, Karen M. Anderson und Isabelle Schulze, 555-604. Oxford: Oxford University Press.

Streeck, Wolfgang. 2003. No Longer the Century of Corporatism. Das Ende des "Bündnisses für Arbeit”. MPIfG Working Paper 03/4. Köln: Max-Planck-Institut für Gesellschaftsforschung.

Swenson, Peter A. 2004. Capitalists against Markets. The Making of Labor Markets and Welfare States in the United States and Sweden. New York: Oxford University Press.

Tálos, Emmerich. 2005. "Sozialpolitik neu". Eine Bilanz von fünf Jahren ÖVP/FPÖRegierung in Österreich. Zeitschrift für Sozialreform 51:273-297.

Trampusch, Christine. 2004a. Von Verbänden zu Parteien. Der Elitenwechsel in der Sozialpolitik. MPIfG Discussion Paper 04/3. Köln: Max-Planck-Institut für Gesellschaftsforschung.

Trampusch, Christine. 2004b. Das Scheitern der Politikwissenschaft am Bündnis für Arbeit. Eine Kritik an der Problemlösungsliteratur über das Bündnis für Arbeit. Politische Vierteljahresschrift 45:541-562.

Trampusch, Christine. 2007. Industrial Relations as a Source of Solidarity in Times of Welfare State Retrenchment. Journal of Social Policy 36:197-215.

Tsebelis, George. 1995. Decision Making in Political Systems. Veto Players in Presidentialism, Parliamentarism, Multicameralism and Multipartyism. British Journal of Political Science 25:289-325.

Tsebelis, George. 2002. Veto Players. How Political Institutions Work. New York/Princeton/Woodstock: Princeton University Press. 
Visser, Jelle, und Anton Hemerijck. 1998. Ein holländisches Wunder? Reform des Sozialstaates und Beschäftigungswachstum in den Niederlanden. Frankfurt a.M./New York: Campus Verlag.

Wiß, Tobias. 2011. Der Wandel der Alterssicherung in Deutschland. Die Rolle der Sozialpartner. Wiesbaden: VS Verlag für Sozialwissenschaften.

\section{Autorenangaben:}

Dr. Tobias Wiß,

Institut für Gesellschafts- und Sozialpolitik, Johannes Kepler Universität Linz, Österreich, tobias.wiss@gmx.de 